\title{
PARP1 might enhance the therapeutic effect of tetrahydroxystilbene glucoside in traumatic brain injury via inhibition of Ras/JNK signalling pathway
}

\author{
Yiqiang $\mathrm{CaO}^{1}$, Yu Chen ${ }^{2}$, Fei Wang ${ }^{1}$, Yonggang Wang ${ }^{1}$, Jiang Long ${ }^{1}$ \\ ${ }^{1}$ Department of Neurosurgery, the First Affiliated Hospital of Kunming Medical University, Kunming, China, ${ }^{2}$ Department of \\ Neurosurgery, the First Affiliated Hospital of Zhengzhou University, Zhengzhou, China
}

\begin{abstract}
Trauma is the main cause of death for people aged 1-45, and among them, traumatic brain injury (TBI) is the major condition, which causes over 50,000 deaths each year and costs over 80 billion per year. Tetrahydroxystilbene glucoside (TSG) is the active ingredient of polygonum multiflorum, a traditional Chinese herbal medicine, which presented multiple pharmacological effects, including antioxidative, anti-inflammatory, reducing blood fat and neuroprotection effects. However, the effect of TSG in promoting the recovery of the nerve system after TBI is not fully understood. PARP1 is a key enzyme in repair of the damage in DNA, which is activated by binding to DNA breaks, initiating both single-strand and double-strand DNA break repair. And we thought that overexpression of TSG might enhance the effect of TSG in $T B I$ treatment. In this study, we firstly detected the oxidative stress response related molecules in serum samples of TBI patients and a TBI mice model, and found that oxidative stress response was activated after TBI, and TSG would reduce this effect. We further noticed that inflammation related molecules presented a similar trend with oxidative stress response related molecules. These results indicated that inflammatory response and oxidative stress processes were both activated after TBI, and reduced after TSG treatment. We further detected that the apoptosis related proteins and anti-oxidative proteins were increased after TSG treatment, and these effects were enlarged after overexpression of PARP1. We further noticed that these effects might be mediated by inhibition of the Ras/JNK signalling pathway. Thus, we thought overexpression of PARP1 might enhance the therapeutic effect of TSG in TBI treatment.
\end{abstract}

Key words: PARP1, tetrahydroxystilbene glucoside, traumatic brain injury, Ras/JNK signalling pathway.

\section{Introduction}

Traumatic brain injury (TBI) is one of the major causes of death and disability around the world. According to a previous study, the annual incidence of TBI is 10 million cases of death with the prevalence of 3.17 million of long-term TBI-induced disability, and it causes an economic burden of billions of dollars $[15,24]$. These disabilities are not only the mechanical damage caused by the initial injury, but are also caused by subsequent cellular and molecular damage in following days [29]. Tetrahydroxystilbene glucoside (TSG) is the main component of polygonum multiflorum, performs strong anti-oxidative and free radical-scavenging activities, and presented a variety of functions in previous studies, especially

\section{Communicating author}

Jiang Long, Department of Neurosurgery, the First Affiliated Hospital of Kunming Medical University, No. 295 Xichang Road, Wuhua District, Kunming, 650032, China, e-mail: Jiang_long2528@outlook.com 
in cardiovascular protection and neuroprotection [37]. PARP1 is the first member of the PARP family, which was identified in 1987 [1]. PARP1 could detect the breaks in DNA strand and activate cellular DNA damage response through the activation of downstream biological process, including DNA damage repair, epigenetic regulation, transcription, apoptosis, and mRNA metabolism [20].

In the present study, we firstly noticed that the oxidative stress response was activated in TBI patients and a TBI mice model, and TSG treatment would reduce this effect. Besides, we also noticed that the expression of inflammatory response related molecules was increased and TSG treatment would also decrease the expression of these molecules. Besides, the expression of apoptosis related proteins and anti-oxidative enzymes was increased after TSG treatment, and these effects might be mediated by inhibition of the Ras/JNK signalling pathway, while overexpression of PARP1 would enlarge these effects and inhibition of PARP1 expression would reduce these effects. According to these results, we thought that overexpression of PARP1 might be a new therapeutic target in TBI treatment.

\section{Material and methods}

NOS (ab106535, 30), NOX4 (ab154244, 67), Catalase (ab16731, 60), TRX (ab26320, 12), Bcl-2 (ab182858, 26), Cleaved Caspase-3 (ab2302, 17), Caspase-3 (ab13847, 17), Caspase-9 (ab202068, 46), Rho (ab40673, 21), Ras (ab52939, 21), Rac1 (ab33186, 21), p-MEKK1 (ab138662, 164), MEKK1 (ab212601, 164), p-MKK4 (ab52958, 44), MKK4 (ab33912, 44), JNK (ab208035, 48) and p-JNK $(a b 219584,48)$ were purchased from Abcam. ROS/ Superoxide Detection Assay (ab ab186027), Superoxide Dismutase 1 ELISA (ab119520), Lipid Peroxidation (MDA) Assay (ab233471) and NADP/NADPH Assay (ab65349) were purchased from Abcam.

\section{Vector construction}

Full length of PARP1 cDNA was cloned with the PCR method using the following primer: Forward: 5'-ACGCACAATGCCTATGAC-3', Reverse: 5'-CCA GCGGAACCTCTACAC-3'. pLenti-CMV-GFP blank vector was digested with BamHI (R3136S, NEB) and Sall (R3138S, NEB), and connected with PARP1 CDNA using T4 ligase (M0201S, NEB). PARP1 knockdown vector was constructed according to the previous protocol [17]. Briefly, lentiviral CRISPR plasmid was firstly digested with BsmBI (R0580S, NEB), and oligos were composed with the following primer: Forward: 5'-CACCGTTCTAGTCGCCCATGTTTGA-3', Reverse: 5'-CTCAAACATGGGCGACTCGAACAA-3'. Then, PARP1 knockdown vector was obtained using Quick Ligase (NEB M2200S). After vectors were constructed, vectors were transfected into 293T cells (CRL-11268, ATCC) to construct lentiviral vector after being incubated for $48 \mathrm{~h}$, lentiviral vectors were injected into the mice model to construct a PARP1 overexpression mice model and PARP1 knockdown mice model.

\section{Animal model}

20 C57BL mice aged 5-8 weeks old and weighing 24.5-26.2 g were purchased from the Experimental Animal Centre of the Third Military Medical University (Chongqing, China). Animals were housed at $26^{\circ} \mathrm{C}, 55 \%$ humidity atmosphere with $12 \mathrm{~h}$ light/ $12 \mathrm{~h}$ dark cycle. Food and water were freely available. And mice were divided into four groups: $\mathrm{CCl}$ (Controlled Cortical Impact) group (CG, $n=5$ ), CCl group combined with TSG treatment group (CT, $n=5), \mathrm{CCl}$ group combined with TSG treatment and PARP1 inhibition group $(\mathrm{Cl}, n=5)$ and $\mathrm{CCl}$ group combined with TSG treatment and PARP1 overexpression group $(\mathrm{CO}, n=5)$. Mice in the $\mathrm{CCl}$ group were used as a control group.

\section{$\mathrm{CCl}$ model construction}

The $\mathrm{CCl}$ model was constructed according to a previous study [30]. Briefly, mice were firstly anesthetized with $4 \%$ isoflurane inhalation. And $\mathrm{CCl}$ was performed after midline incision and craniotomy with a fabricated impactor (L. Kopacz, Germany; tip diameter, $3 \mathrm{~mm}$; impact velocity, $8 \mathrm{~m} / \mathrm{sec}$; impact duration, $150 \mathrm{~ms}$; impact depth, $1 \mathrm{~mm}$ ). Then, craniotomy and skin were closed and animals were kept as previously described, and treated with $12 \mathrm{mg} / \mathrm{kg}$ TSG for seven days by an intraperitoneal injection [39]. After treatment, brain tissues and serum samples were collected to perform the following experiments.

\section{Ethical statement}

This study was carried out in strict accordance with the recommendations in the Guide for the Care and Use of Laboratory Animals of the National Institutes of Health (USA). The protocol was approved by the Committee on the Ethics of Animal Experiments of the First Affiliated Hospital of Kunming 
Medical University. All experiments were conducted in accordance with the Declaration of Helsinki and were approved by the Ethics Committee of the First Affiliated Hospital of Kunming Medical University. All study participants gave written informed consent.

\section{RNA extraction and reverse transcription}

RNA extraction was performed according to the protocol (CW0560S, CWbio). Briefly, tissues were lysed with lysis buffer and incubated at room temperature for $5 \mathrm{~min}$. After being centrifuged at 12,000 rpm for 5 min and mixed with $70 \%$ ethanol, the mixture was removed into an absorption tube. After being washed with washing buffer, RNAs were eluted from the tube using elution buffer. Then, reverse transcription was performed according to the protocol (CW0741, CWbio). Briefly, the reaction mixture was composed as recommended, and incubated at $42^{\circ} \mathrm{C}$ for $30 \mathrm{~min}$ followed with incubation at $85^{\circ} \mathrm{C}$ for $5 \mathrm{~min}$. The cDNAs were stored at $-20^{\circ} \mathrm{C}$ until the following experiments.

\section{Real-time quantitative polymerase chain reaction ( $q P C R$ )}

qPCR was performed according to the protocol (CW0957, CWbio). Briefly, the reaction mixture was composed as recommended. qPCR was performed using the following primers: IFN- $\gamma$ : Forward: 5'-GCTCTGAGACAATGAACGCT-3', Reverse: 5'-AAAGAGATAATCTGGCTCTGC-3'; IL-10: Forward: 5'-TGTGTCAGCCCTCAGAGTAC-3', Reverse: 5'-CACTGACACTTCGCACAA-3'; IL-1 $\beta$ : Forward: 5'-GTGCTGTCGGACCCATATGAG-3', Reverse: 5'-CAGGAAGACAGGCTTGTGCTC-3'; IL-6: Forward: 5'-TCGTGGAAATGAGAAAAGAGTTG-3', Reverse: 5'-TATGCTTAGGCATAACGCACTAGA-3'. And the reaction steps were set up as follows: pre-degeneration at $95^{\circ} \mathrm{C}$ for $10 \mathrm{~min}$, these two steps repeated for 40 cycles: degeneration at $95^{\circ} \mathrm{C}$ for $15 \mathrm{~s}$, extend $60^{\circ} \mathrm{C}$ for $1 \mathrm{~min}$, fusion curve analysis: $95^{\circ} \mathrm{C}$ for $15 \mathrm{~s}, 60^{\circ} \mathrm{C}$ for $1 \mathrm{~min}$, $95^{\circ} \mathrm{C}$ for $15 \mathrm{~s}$ and $60^{\circ} \mathrm{C}$ for $15 \mathrm{~s}$. GAPDH was used as an internal control, and data were analysed using $2^{-\Delta \Delta C t}$ method according to the previous study [27].

\section{Western blotting analysis}

Brain tissues were lysed with lysis buffer (8 M Urea, $50 \mathrm{mM}$ IAA, $10 \mathrm{mM}$ DTT and proteinase inhibitor cocktail). After being centrifuged at 12000 rpm for $10 \mathrm{~min}$, proteins were collected and concentration of proteins was determined using BCA assay. $60 \mu \mathrm{g}$ pro- tein samples were separated using 10\% SDS-PAGE electrophoresis, after that proteins were transferred onto a nitrocellulose membrane using a semi-dry trans blotter. Then, membranes were blocked with 5\% skim milk followed by incubation with primary antibodies ( $1: 2000)$ overnight at $4^{\circ} \mathrm{C}$. After incubation, membranes were incubated with secondary antibody (1 : 5000) for $1 \mathrm{~h}$ at room temperature. Grey value of each protein was detected using chemiluminescent immunoassay, and GAPDH was used as an internal control.

\section{Enzyme-linked immunosorbent assay (ELISA)}

Serum samples collected from mice and male TBI patients were collected to perform the ELISA experiments $(n=20)$. The average age of TBI patients is 49.5 \pm 8.5 years old, and the average age of the male normal control group $(n=20)$ is $46.7 \pm 7.7$ years old. Patients with a definite history of craniocerebral trauma and without other operation history recently were brought into this experiment. Patients with severe dysfunction in the major organ (such as the heart, liver, lung and kidney), patients with injury in other parts of the body and patients with a previous history of craniocerebral trauma were excluded from this experiment. And ELISA experiments were performed according to the protocol. Briefly, serum samples, standards and reaction buffer were added into each well, and incubated at room temperature for $3 \mathrm{~h}$, after being washed with washing buffer, TMB substrate solution was added into each well and incubated at room temperature for $10 \mathrm{~min}$. After stop solution was added, the optical density was detected at an appropriate wave length using a spectrophotometer (Multiskan GO, Thermo). And data were normalized with the corresponding control group.

\section{Statistical analysis}

Data were presented as mean \pm SD, each experiment was repeated for three times independently. One-way ANOVA was used to compare the differences between groups, $p<0.05$ was set as a statistical difference.

\section{Results}

\section{Concentration of oxidative stress response related molecules in serum samples of $\mathrm{TBI}$ patients}

As shown in Figure 1, the relative concentrations of ROS in normal patients and TBI patients were 

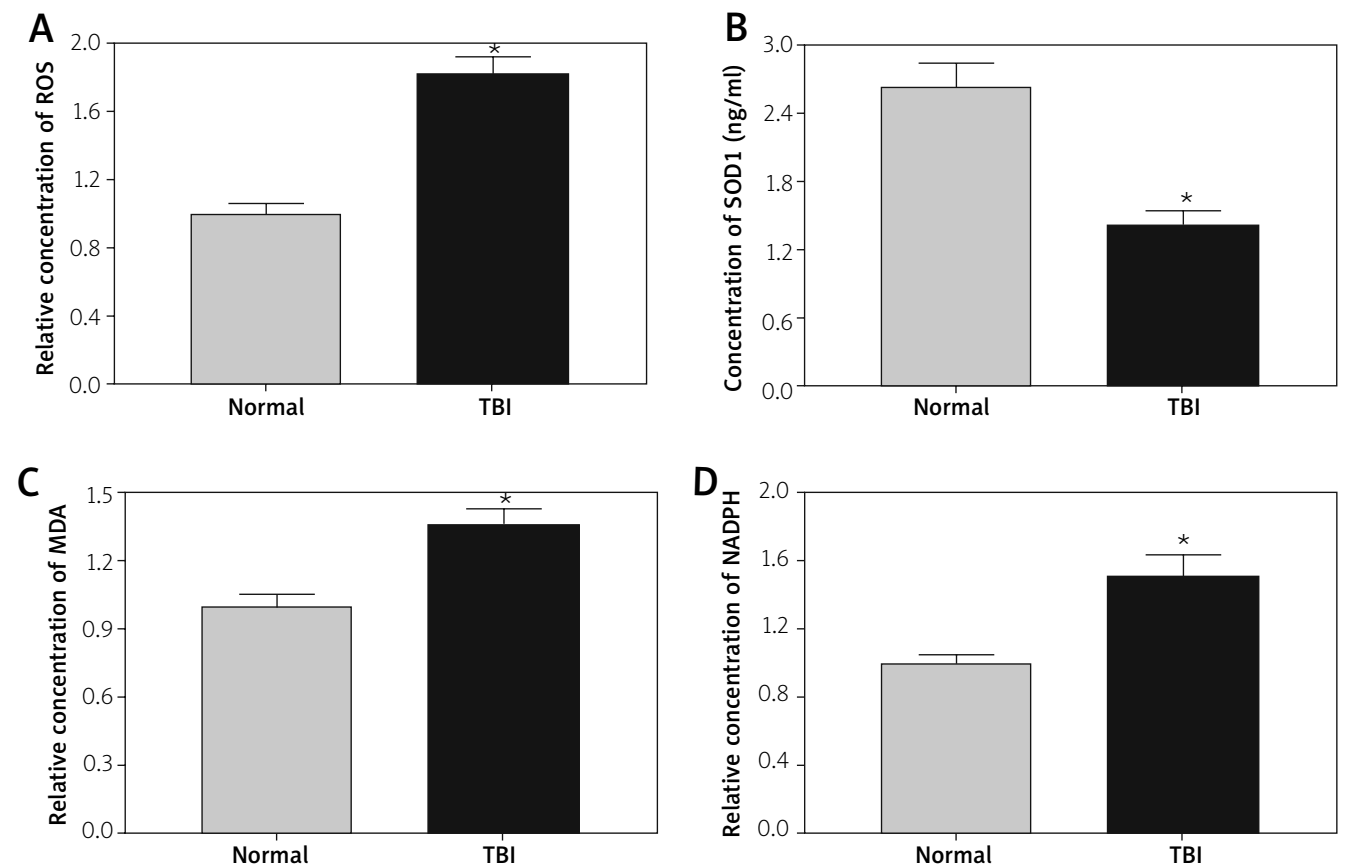

Fig. 1. Concentration of oxidative stress response related molecules in serum samples of TBI patients. A) Concentration of ROS in serum samples. B) Concentration of SOD1 in serum samples. C) Concentration of MDA in serum samples. D) Expression of NADPH in serum samples. Data were presented as mean \pm SD. Each experiment was repeated for three times independently. ${ }^{*} p<0.05$ compared with the CG group, ${ }^{\#} p<0.05$ compared with the CT group.

$1.00 \pm 0.06,1.82 \pm 0.10$, respectively. The concentrations of SOD 1 in these groups were $2.64 \pm 0.19,1.42$ \pm 0.12 , respectively. The relative concentrations of MDA in these groups were $1.00 \pm 0.05,1.36 \pm 0.07$, respectively. The relative concentrations of NADPH in these groups were $1.00 \pm 0.05,1.51 \pm 0.13$, respectively. Relative concentrations of ROS, MDA and NADPH were significantly increased in serum samples of TBI patients, and the concentration of SOD1 was significantly decreased in TBI patients, indicating that TBI patients were under an oxidative stress status.

\section{Concentration of oxidative stress response related molecules in serum samples of mice}

As shown in Figure 2, the concentrations of ROS in CG, CT, CO and $\mathrm{Cl}$ groups were $1.00 \pm 0.08,0.71$ $\pm 0.06,0.44 \pm 0.04$ and $1.24 \pm 0.11$, respectively. The relative concentrations of ROS in $\mathrm{CT}$ and $\mathrm{CO}$ groups were significantly decreased and in the $\mathrm{Cl}$ group it was significantly increased compared with the CG group $(p<0.05)$, and was significantly decreased in the CO group and significantly increased in the $\mathrm{Cl}$ group compared with the CT group $(p<0.05)$. The concentrations of SDO1 in these groups were 0.72 $\pm 0.07,1.15 \pm 0.12,1.56 \pm 0.14$ and $0.77 \pm 0.06 \mathrm{ng} / \mathrm{ml}$, respectively. The relative concentrations of SOD1 in CT and CO groups were significantly decreased and in the $\mathrm{Cl}$ group it was significantly increased compared with the CG group ( $p<0.05)$, and was significantly decreased in the CO group compared with the CT group $(p<0.05)$. The relative concentrations of MDA in these groups were $1.00 \pm 0.07,0.68 \pm 0.05$, $0.36 \pm 0.02$ and $0.84 \pm 0.07$, respectively. The relative concentration of MDA presented a similar trend with ROS. The concentrations of NADPH in these groups were $1.00 \pm 0.08,1.35 \pm 0.14,1.88 \pm 0.16$ and 0.94 \pm 0.07 , respectively. Change in the concentration of NADPH presented a similar trend with SOD1.

\section{Expression of inflammation response related genes in brain tissues}

As shown in Figure 3, the expressions of inflammation response related genes in brain tissues were 
A
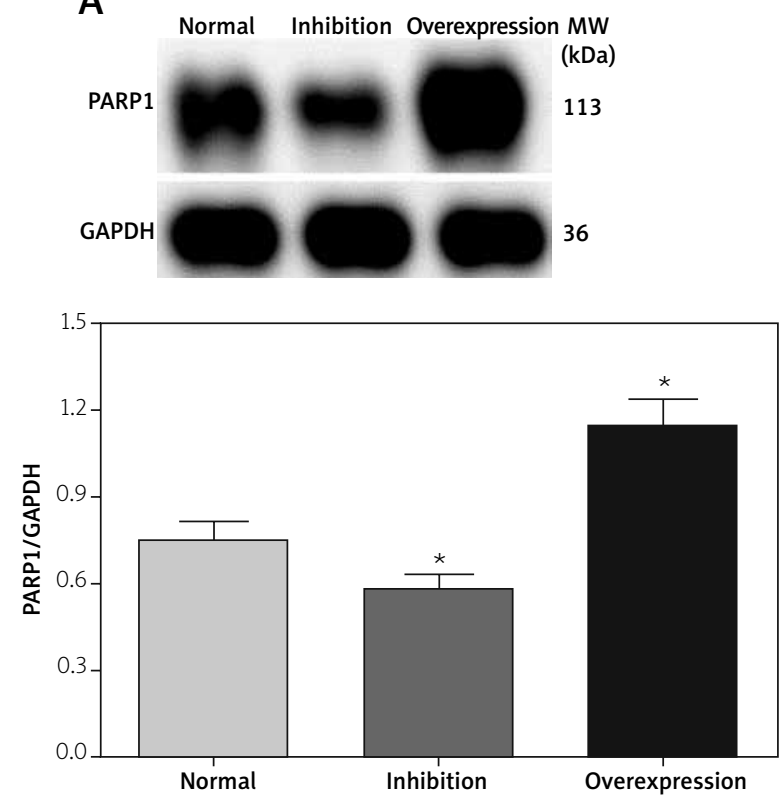
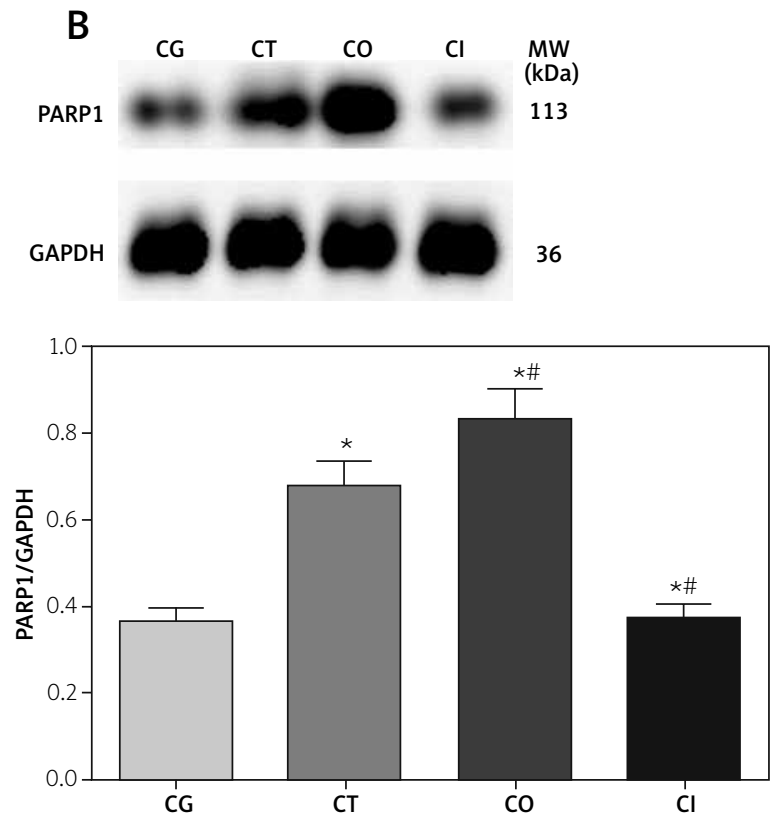

Fig. 2. Expression of PARP1 in brain tissues of a mice model. A) Expression of PARP1 in brain tissue of a mice model without TSG treatment. B) Expression of PARP1 in brain tissue of mice model after TSG treatment. Data was presented as mean \pm SD. Each experiment was repeated for three times independently. ${ }^{*} p<0.05$ compared with the CG group, ${ }^{*} p<0.05$ compared with the CT group.

detected using the qPCR method. The expressions of IFN- $\gamma$ in CG, CT, CO and Cl groups were $0.52 \pm 0.04$, $0.87 \pm 0.06,1.24 \pm 0.11$ and $0.56 \pm 0.04$, respectively. The expression of IFN- $\gamma$ was significantly increased in the CT and CO groups compared with the CG group $(p<0.05)$, and significantly increased in the $\mathrm{CO}$ group and significantly decreased in the $\mathrm{Cl}$ group $(p<0.05)$ compared with the CT group. The expressions of IL-1 $\beta$ in these groups were $1.27 \pm 0.13,0.92$ $\pm 0.10,0.61 \pm 0.05$ and $1.19 \pm 0.11$, respectively. The expression of IL-1 $\beta$ was significantly decreased in the CT and CO groups compared with the CG group ( $p<0.05)$, and significantly decreased in the $\mathrm{CO}$ group and significantly increased in the $\mathrm{Cl}$ group ( $p<0.05)$ compared with the CT group. The expressions of IL- 6 in these groups were $1.34 \pm 0.14,1.05$ $\pm 0.09,0.74 \pm 0.06$ and $1.28 \pm 0.12$, respectively. The expression of IL-1 $\beta$ was significantly decreased in the CT and CO group compared with the CG group $(p<0.05)$, and significantly decreased in the CO group $(p<0.05)$ compared with the CT group. The expressions of IL-10 in these groups were $0.64 \pm 0.05$, $0.98 \pm 0.09,1.47 \pm 0.13$ and $0.72 \pm 0.07$, respectively. The change in the expression of $\mathrm{IL}-10$ presented a similar trend with interferon $\gamma($ IFN- $\gamma)$.

\section{Expression of PARP1 in each group of the mice model}

As shown in Figure 4, the expression levels of PARP1 in brain tissues of normal, PARP1 inhibition and PARP1 overexpression group of mice without TSG treatment were $0.75 \pm 0.06,0.58 \pm 0.05$ and $1.14 \pm 0.10$, respectively. The expression of PARP1 was significantly decreased in the inhibition group $(p<0.05)$ and significantly increased in the overexpression group $(p<0.05)$. And after TSG treatment, the expressions of PARP1 in CG, CT, Cl and CO groups were $0.37 \pm 0.03$, $0.68 \pm 0.06,0.83 \pm 0.07$ and $0.37 \pm 0.03$, respectively. The expression of PARP1 was significantly increased in the CT and CO group ( $p<0.05)$ and significantly decreased in the $\mathrm{Cl}$ group $(p<0.05)$ compared with the CG group, and significantly increased in the CO group $(p<0.05)$ and significantly decreased in the $\mathrm{Cl}$ group $(p<0.05)$ compared with the CT group.

\section{Expression of apoptosis related proteins and anti-oxidative enzymes in each group of the mice model}

As shown in Figure 5, the expressions of NOS in CG, $\mathrm{CT}, \mathrm{CO}$ and $\mathrm{Cl}$ group were $5.63 \pm 0.47,4.22 \pm 0.35,2.47$ 
A

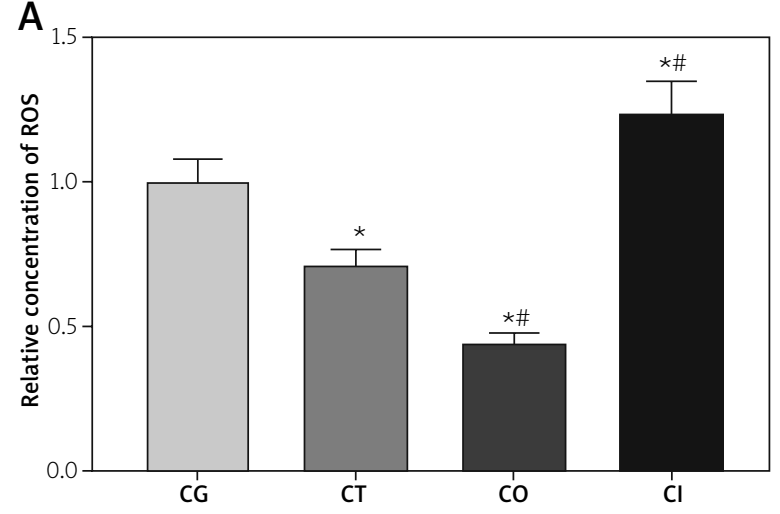

C

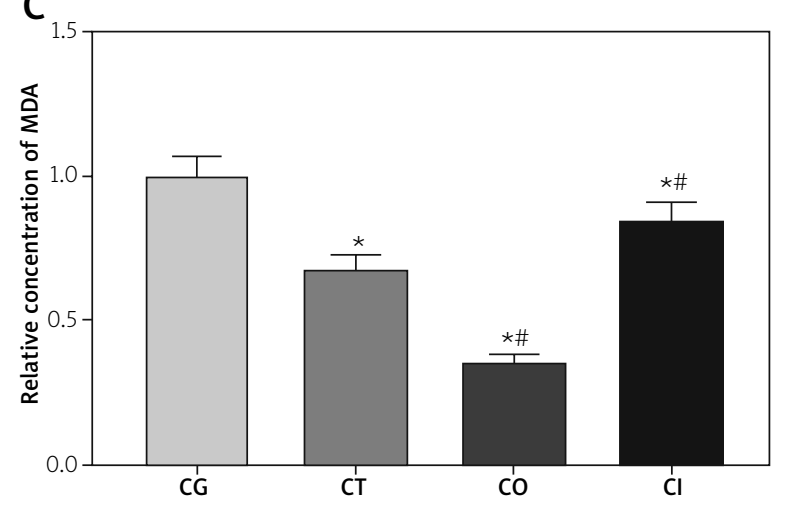

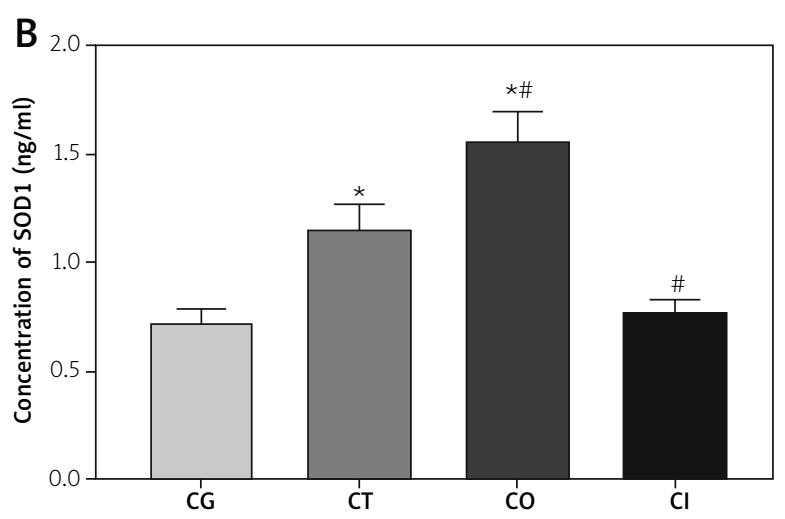

D

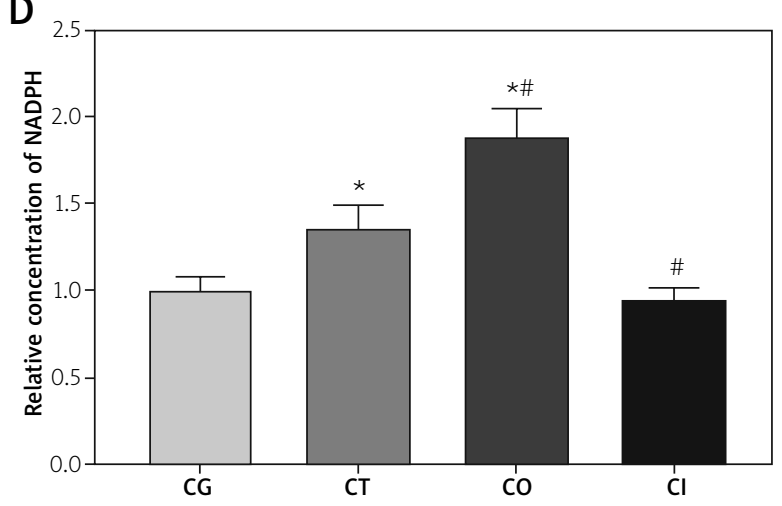

Fig. 3. Concentration of oxidative stress response related molecules in serum samples of a mice model. A) Concentration of ROS in serum samples. B) Concentration of SOD1 in serum samples. C) Concentration of MDA in serum samples. D) Expression of NADPH in serum samples. Data were presented as mean \pm SD. Each experiment was repeated for three times independently. ${ }^{*} p<0.05$ compared with the CG group, ${ }^{\#} p<0.05$ compared with the CT group.

\pm 0.21 and $5.60 \pm 0.47$, respectively. The expression of NOS was significantly decreased in CT and CO groups compared with the CG group $(p<0.05)$, and significantly decreased in the $\mathrm{CO}$ group and significantly increased in the $\mathrm{Cl}$ group $(p<0.05)$ compared with the CT group. The expressions of NOX4 in these groups were $1.69 \pm 0.14$, $1.69 \pm 0.14,1.35 \pm 0.11$ and $2.14 \pm 0.18$, respectively. The expression of NOX4 was significantly decreased in the $\mathrm{CO}$ group and significantly increased in the $\mathrm{Cl}$ group $(p<$ 0.05) compared with the CG and CT group. The expressions of catalase in these groups were $1.14 \pm 0.10$, $1.84 \pm 0.15,1.91 \pm 0.16$ and $1.19 \pm 0.10$, respectively. The expression of catalase was significantly increased in the CT and CO group $(p<0.05)$ compared with the CG group, and significantly decreased in the $\mathrm{Cl}$ group $(p<0.05)$ compared with the CT group. The expressions of TRX in these groups were $0.62 \pm 0.05,0.92 \pm 0.08$, $1.39 \pm 0.12$ and $0.27 \pm 0.02$, respectively. The change in expression of TRX presented a similar trend with NOS. The expressions of Bcl-2 in these groups were $1.04 \pm 0.09,1.54 \pm 0.13,1.87 \pm 0.16$ and $1.03 \pm 0.09$, respectively. The expression of $\mathrm{BCl}-2$ was significantly increased in the CT and CO group $(p<0.05)$ compared with the CG group, and significantly increased in the $\mathrm{CO}$ group and significantly decreased in the $\mathrm{Cl}$ group ( $p$ $<0.05$ ) compared with the CT group. The ratio of activated caspase-3/caspase- 3 in these groups were 1.51 $\pm 0.13,1.17 \pm 0.10,0.56 \pm 0.05$ and $1.53 \pm 0.13$, respectively. The expressions of caspase- 9 in these groups were $1.19 \pm 0.10,0.69 \pm 0.06,0.33 \pm 0.03$ and $1.13 \pm 0.09$, respectively. The change in expression of caspase- 3 and caspase- 9 was significantly decreased in the $\mathrm{CT}$ and $\mathrm{CO}$ group $(p<0.05)$ compared with the CG group, and significantly decreased in the $\mathrm{CO}$ group and significantly increased in the $\mathrm{Cl}$ group $(p<0.05)$ compared with the CT group. 
A

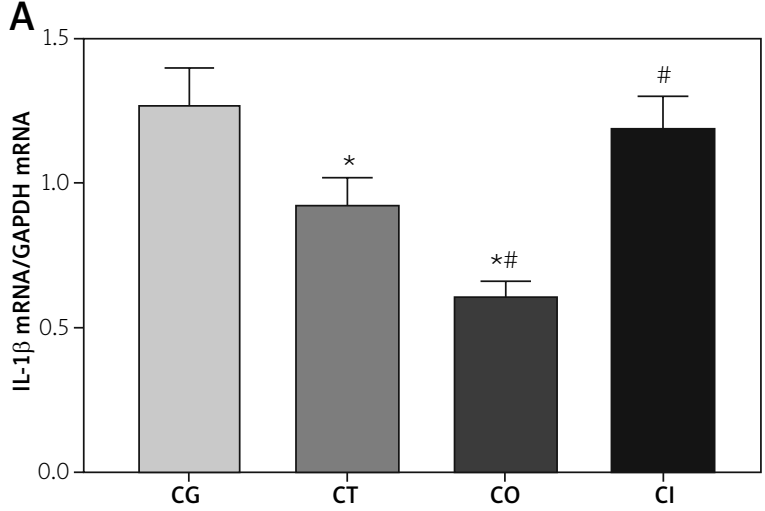

C

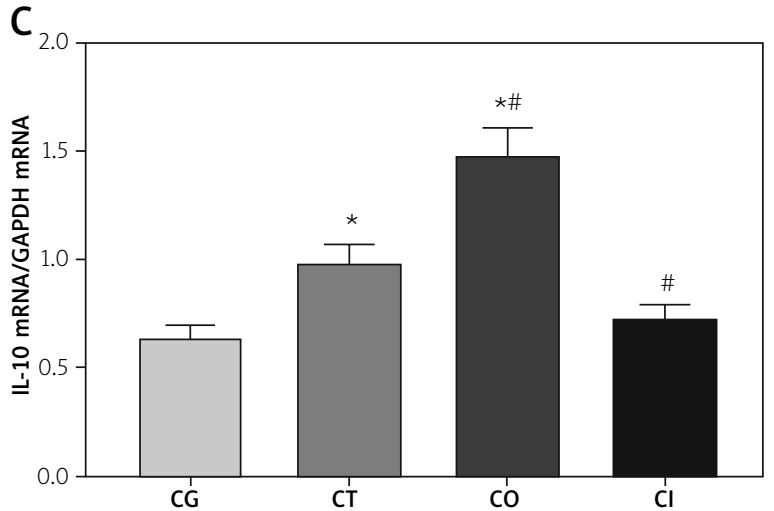

B

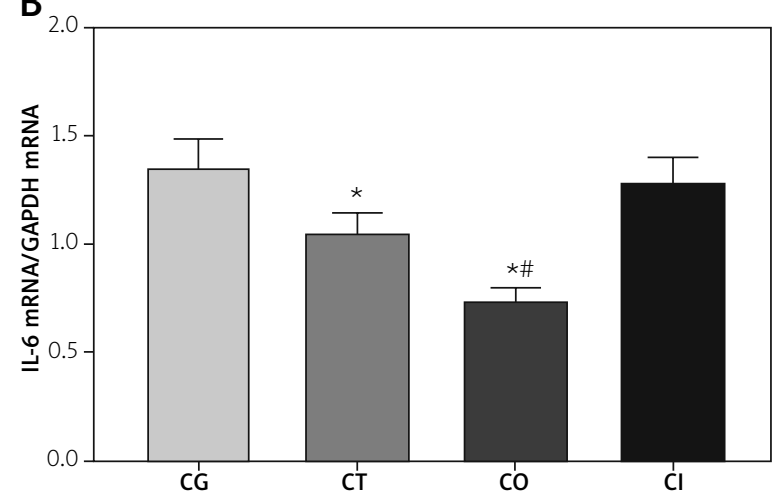

D

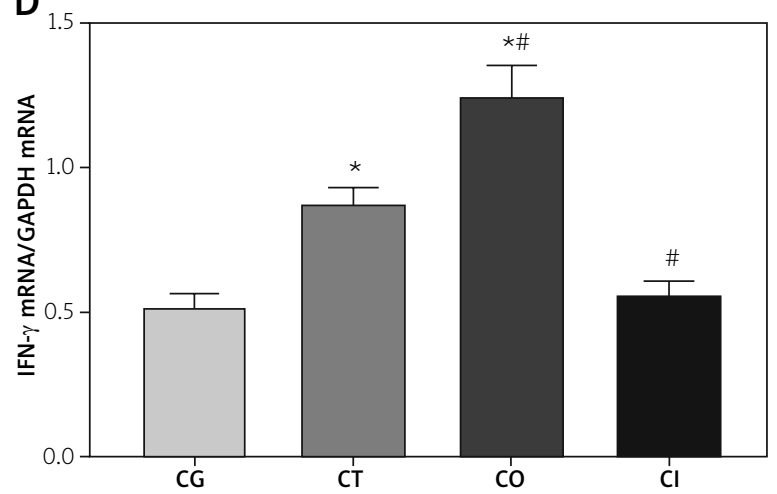

Fig. 4. Expression of inflammatory response related genes in brain tissues of a mice model. A) Expression of IL-1 $\beta$ in brain tissue of a mice model. B) Expression of IL- 6 in brain tissue of a mice model. C) Expression of IL-10 in brain tissue of a mice model. D) Expression of IFN- $\gamma$ in brain tissue of a mice model. Data were presented as mean \pm SD. Each experiment was repeated for three times independently. ${ }^{*} p<0.05$ compared with the CG group, ${ }^{*} p<0.05$ compared with the CT group.

\section{Activation of Ras/JNK signalling pathway in each group of the mice model}

As shown in Figure 6, the activation of Ras/JNK signalling pathway was detected using western blotting analysis. The expressions of Rho in CG, CT, CO and $\mathrm{Cl}$ groups were $0.92 \pm 0.08,0.84 \pm 0.07,0.32 \pm 0.03$ and $1.16 \pm 0.10$, respectively. The expression of Rho was significantly decreased in the CO group $(p<0.05)$ and significantly increased in the $\mathrm{Cl}$ group $(p<0.05) \mathrm{com}$ pared with the CG and CT group. The expressions of Ras in these groups were $0.75 \pm 0.06,0.47 \pm 0.04,0.20$ \pm 0.02 and $1.00 \pm 0.08$, respectively. The expression of Ras was significantly decreased in the $\mathrm{CT}$ and $\mathrm{CO}$ group $(p<0.05)$ compared with the CG group, and significantly increased in the Cl group $(p<0.05)$ compared with the CG group. And it was significantly decreased in the $\mathrm{CO}$ group $(p<0.05)$ and significantly increased in the $\mathrm{Cl}$ group $(p<0.05)$ compared with the $\mathrm{CT}$ group. The expressions of Rac1 in these groups were $1.17 \pm 0.10,0.98 \pm 0.08$, $0.60 \pm 0.05$ and $1.34 \pm 0.11$. The expression of Rac1 was significantly decreased in the CO group $(p<0.05)$ compared with the CG and CT group, and was significantly increased in the $\mathrm{Cl}$ group $(p<0.05)$ compared with the CT group. The ratios of p-MEKK1/MEKK1 in these groups were $1.60 \pm 0.13,1.69 \pm 0.14,1.30 \pm 0.11$ and 1.95 \pm 0.16 , respectively. The ratio of $\mathrm{p}-\mathrm{MEKK} 1 /$ MEKK 1 was significantly decreased in the CO group $(p<0.05)$ and significantly increased in the $\mathrm{Cl}$ group $(p<0.05) \mathrm{com}$ pared with the CG group, and significantly decreased in the CO group $(p<0.05)$ compared with the CT group. The ratios of $p$-MEK4/MEK4 in these groups were $1.17 \pm 0.10,0.70 \pm 0.06,0.31 \pm 0.03$ and $1.05 \pm 0.09$, respectively. The ratio of $\mathrm{p}-\mathrm{MEK} 4 / \mathrm{MEK} 4$ was significantly decreased in the CT and CO group $(p<0.05)$ 

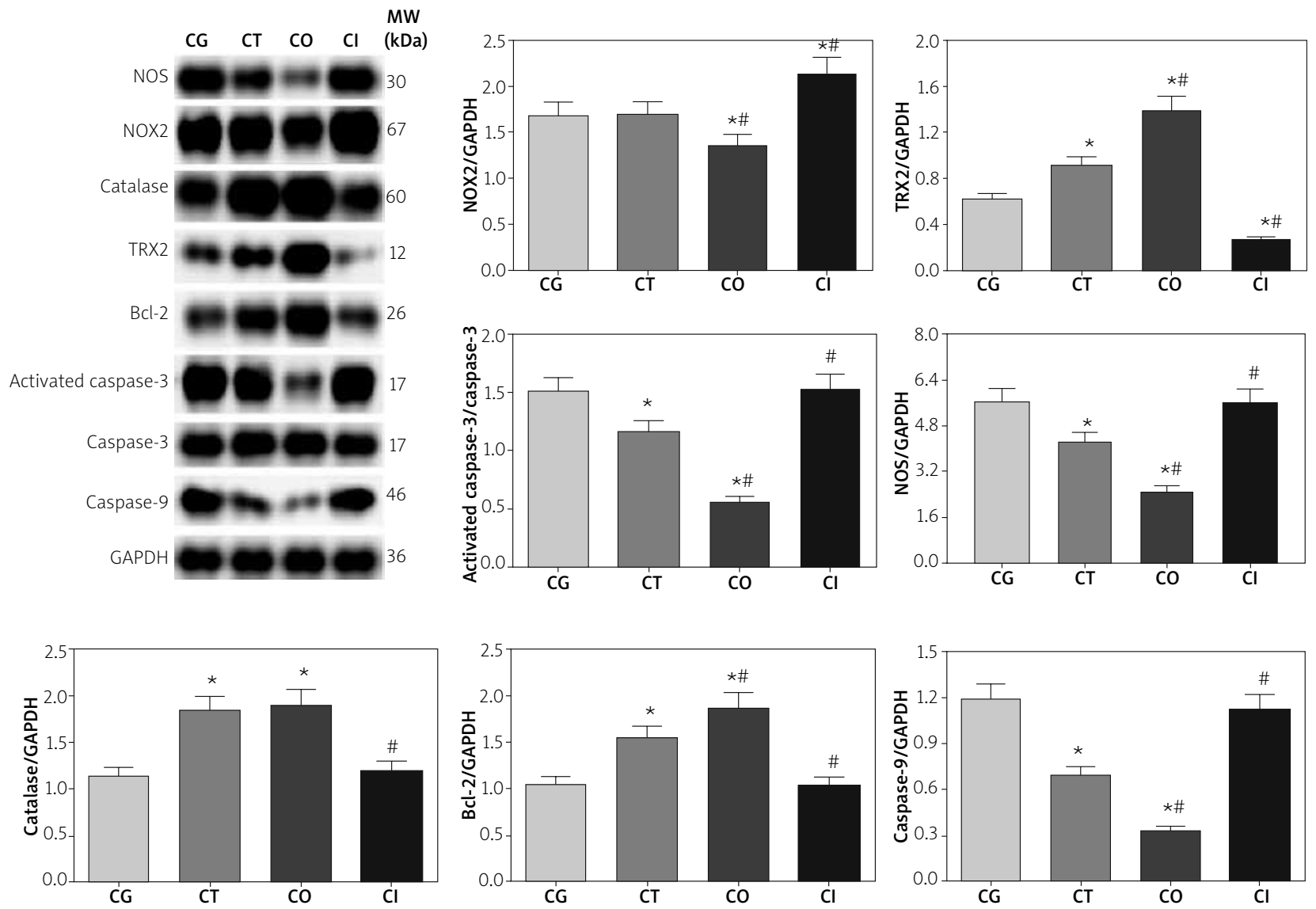

Fig. 5. Expression of apoptosis related molecules and anti-oxidative enzymes in brain tissues of a mice model. Data were presented as mean \pm SD. Each experiment was repeated for three times independently. ${ }^{*} p<0.05$ compared with the CG group, ${ }^{*} p<0.05$ compared with the CT group.

compared with the CG group, and was significantly decreased in the CO group $(p<0.05)$ and significantly increased in the $\mathrm{Cl}$ group $(p<0.05)$ compared with the CT group. The ratios of $p-J N K / J N K$ in these groups were $1.32 \pm 0.11,1.25 \pm 0.10,0.91 \pm 0.08$ and $1.75 \pm 0.15$, respectively. The ratio of $p$-JNK/JNK was significantly decreased in the CO group $(p<0.05)$ and significantly increased in the $\mathrm{Cl}$ group $(p<0.05) \mathrm{com}$ pared with the CG and CT group.

\section{Discussion}

Traumatic brain injury (TBI) is a great threaten in global health care, with more than 27 million new cases each year [11]. In the process of TBI, long axons are especially susceptible to compression and tension, resulting in axonal injury. Besides, inflammation and degeneration process in neurons lasts for years after TBI, causing chronic symptoms in $\mathrm{TBI}$ patients [13]. There is plenty of treatments for $\mathrm{TBI}$ nowadays, including traditional medicine treatment, transplanting neural stem cells (NSCs) and bone marrow-derived mesenchymal stem cells [14]. Among them, tetrahydroxystilbene glucoside (TSG) is extracted from traditional Chinese medicine, and has anti-atherosclerosis, hepatoprotective and hypolipidemic effects. However, the effect of TSG in TBI was not clear. Poly-ADP-ribose polymerase 1 (PARP1) is a multi-function enzyme, regulates intracellular processes, including DNA repair, signalling transduction and metabolism via interaction with other proteins and DNA, promoting DNA repair [36].

Rho and Rac1 are family members of GTPases, directly regulate the function of cellular process [23]. Binding of Rho, Rac1 or Cdc43 to p21-binding domain (PBD) of group I PAKs induces the phosphory- 

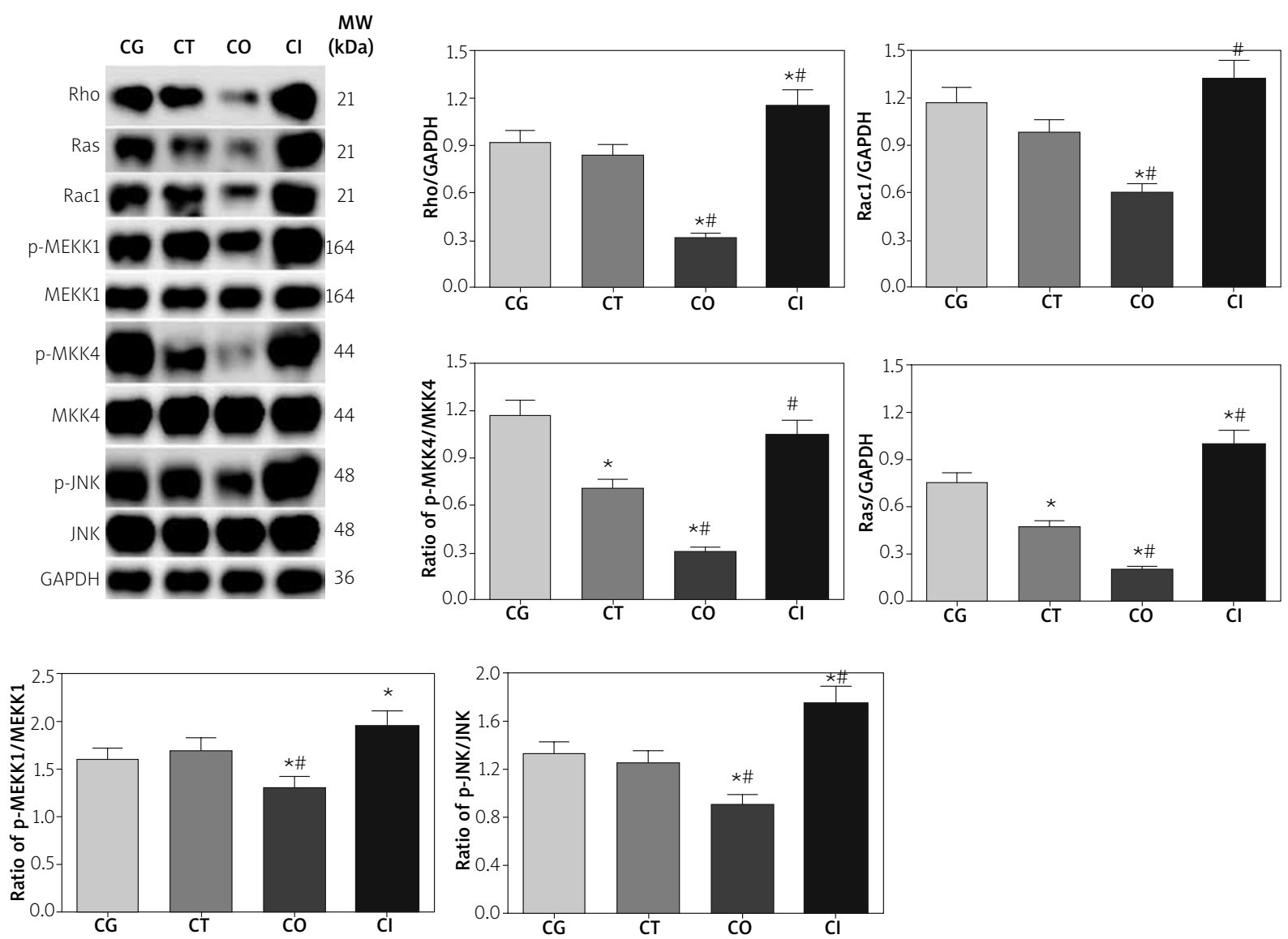

Fig. 6. Activation of the Ras/JNK signalling pathway in brain tissues of a mice model. Data were presented as mean \pm SD. Each experiment was repeated for three times independently. ${ }^{*} p<0.05$ compared with the CG group, ${ }^{*} p<0.05$ compared with the CT group.

lation and activation of multiple downstream molecules [4], further promotes the polymerization of actin and the reduction in expression of PTEN [19], resulting in the activation of the target effector of MAPK, such as ERK and JNK [40]. Ras/mitogen-activated protein kinase (MAPK) signalling pathway regulates the cell proliferation, and has been considered as a key molecular pathway in development of disease both in experimental models and humans $[2,21]$. Ras/MAPK signalling pathway is activated by multiple cytokines, insulin and oxidative stress. Activated Ras would further activate Raf kinase, leading to the phosphorylates of further kinase cascades, MEK1 and MKK4. This process would lead to the phosphorylate of downstream effector molecules, including extracellular signal regulated kinases (ERKS) and c-Jun N-terminal kinase (JNK), regulates the expression of specific genes [33]. However, the effect of JNK in cellular apoptosis process remains controversial. There are studies reporting that TNF- $\alpha$ induced the activation of JNK, which leads to the cellular survival or differentiation via increasing expression of anti-apoptotic genes induced by NF- $\mathrm{KB}$ signalling pathway [8]. But there are also studies indicating that activation of JNK further activates the apoptosis process. The previous study indicated that JNK could directly inhibit the expression of Bcl-2 [38]. Besides, activated JNK were further translocated into mitochondria and induced the release of cytochrome c [6], and this effect might be mediated by activation of caspase cascade [9]. In this study, we found that TSG treatment decreased the expression of p-JNK, p-MKK4, p-MEKK1 and Rho family proteins, indicating that TSG treatment inhibited the activation of Ras/MAPK signalling pathway. We also found that these effects could be enhanced by over- 
expression of PARP1. Considering the former results of our study, we thought that Ras/MAPK/JNK signalling pathway NO is composed by NO synthase and performs a critical role in the central nervous system (CNS), including neurotransmitter, neurogenesis and cerebral blood flow $[25,26]$. NO levels in the damaged cortex were either decreased a few hours after injury or not changed by $72 \mathrm{~h}$, while in cerebellum the levels of NO were increased by $72 \mathrm{~h}$ [41]. The previous study detected the concentration of NO and activity of NOS in TBI site of the mice model, and the concentration of NO presented a significantly increase in injured brain tissue [7]. The activity of NOS in injured brain tissue presented a similar trend with NO [34]. However, the detail mechanism of these changes is not fully understood. In this study, we noticed that the expression of NOS was decreased after TSG treatment, indicating that the injury in brain tissues was recovered by TSG treatment. PARP1 is known as a downstream molecule of NO, overexpression of PARP1 might decrease the expression of NOS in a negative regulation manner, enhance the therapeutic effect of TSG.

Oxidative stress and ROS production were the main causes of cellular damage in the TBI model, the previous study found that the expression of NOX2 was increased with the occurrence of cellular damage, inflammatory and oxidative stress, and these effects might be attenuated after deletion of NOX2 [35]. Superoxide injury is more dependent on NOX signalling pathway according to a previous study, mice with a deficiency of NOX2 in brain tissue had better functional outcomes compared with the normal group [26]. However, we found that TSG treatment alone could not down-regulate the expression of NOX2, and combined with PARP1 overexpression, the expression of NOX2 was significantly decreased, indicating that PARP1 overexpression might enhance the effect of TSG in a TBI model. The anti-oxidative system plays an important role in preventing cellular oxidative stress stimulation. The thioredoxin system is one of the most important anti-oxidative systems in mammalian cells, TRX reductase and NADPH is critical for balancing of cellular redox and cellular anti-oxidants [22]. TRX2 is a protein located in mitochondria and performs an anti-oxidative function via transferring electrons to peroxiredoxins and methionine sulfoxide reductases. A previous study shown that TRX-2 deficiency leads to the accumulation of ROS and cellular apoptosis in cul- tured cells [32]. There is also a study showing that overexpression of TRX2 protects the retinal ganglion neurons against degeneration in experimental models [5]. Besides, catalase plays an important role in response to hydrogen peroxide to ROS [31], catalyses dismutation of $\mathrm{H}_{2} \mathrm{O}_{2}$ into water and molecular oxygen. Besides, catalase could also decompose peroxynitrite and oxidized nitric oxide into nitrogen dioxide. High accumulation of $\mathrm{H}_{2} \mathrm{O}_{2}$ leads to the low level of catalase expression [18]. These effects might induce the activation of diseases related signalling pathways, such as Alzheimer's disease [12]. There is also a study showing that overexpression of catalase using cubosome nanoparticles could prevent the development of neurodegenerative disorders [28]. TSG treatment increases the expression of these two enzymes, and PARP1 overexpression enhances this effect, indicating that the function of anti-oxidative enzyme was recovered, leading to the decreasing of cellular ROS concentration. Oxidative stress after TBI also contributes to the cellular apoptosis. Cellular apoptosis contains two main pathways, intrinsic apoptotic pathway and extrinsic apoptotic pathway. B-cell lymphoma-2 (Bcl-2) family proteins is in the centre of apoptosis regulation through the intrinsic apoptotic pathway, and $\mathrm{BCl}-2$ is the anti-apoptosis molecule of the $\mathrm{Bcl}-2$ family of proteins [3]. The regulation of apoptosis process via $\mathrm{Bcl}-2$ expression is mainly through the MMP, and the loss of MMP would lead to the cellular apoptosis caused by the release of pro-apoptotic proteins, including cytochrome c. Cytochrome c further interacted with APAF1 (apoptotic protease activating factor 1 ), then activated the caspase- 9 from the apoptosome [16]. Activated caspase- 9 further led to the activation of downstream executioner molecules, including caspase-3, 6 and 7, inducing the cellular apoptosis [10]. The expression of $\mathrm{Bcl}-2$ was increased and the expression of caspase cascades was decreased after TSG treatment, indicating that TSG treatment improves the survival of neural cells and PARP1 overexpression would enhance this effect.

\section{Conclusions}

In this study, we noticed that TSG treatment reduces the oxidative stress, inflammatory and apoptosis response in TBI patients and a TBI mice model, and these effects might be mediated by inhibition of the Ras/JNK signalling pathway. PARP1 
overexpression would enhance these effects. The results of our present study indicated that PARP1 might be a therapeutic target of TBI, and we noticed that overexpression of PARP1 presented a promising effect in improving the molecular function in a mice model. However, more experiments on the animal model and TBI patients in our following research are still needed in order to apply this finding in treatment of TBI patients.

\section{Disclosure}

\section{The authors report no conflict of interest.}

\section{References}

1. Alkhatib HM, Chen DF, Cherney B, Bhatia K, Notario V, Giri C, Stein G, Slattery E, Roeder RG, Smulson ME. Cloning and expression of cDNA for human poly(ADP-ribose) polymerase. Proc Natl Acad Sci USA 1987; 84: 1224-1228.

2. Aravalli RN, Cressman EN, Steer CJ. Cellular and molecular mechanisms of hepatocellular carcinoma: an update. Arch Toxicol 2013; 87: 227-247.

3. Ashkenazi A, Fairbrother WJ, Leverson JD, Souers AJ. From basic apoptosis discoveries to advanced selective BCL-2 family inhibitors. Nat Rev Drug Discov 2017; 16: 273-284.

4. Baskaran Y, Ng YW, Selamat W, Ling FT, Manser E. Group I and II mammalian PAKs have different modes of activation by $\mathrm{Cd} c 42$ EMBO Rep 2012; 13: 653-659.

5. Caprioli J1, Munemasa Y, Kwong JM, Piri N. Overexpression of thioredoxins 1 and 2 increases retinal ganglion cell survival after pharmacologically induced oxidative stress, optic nerve transection, and in experimental glaucoma. Trans Am Ophthalmol Soc 2009; 107: 161-165.

6. Chauhan D, Li G, Hideshima T, Podar K, Mitsiades C, Mitsiades N, Munshi N, Kharbanda S, Anderson KC. JNK-dependent release of mitochondrial protein, Smac, during apoptosis in multiple myeloma (MM) cells. J Biol Chem 2003; 278: 1759317596.

7. Cherian L1, Goodman JC, Robertson CS. Brain nitric oxide changes after controlled cortical impact injury in rats. J Neurophysiol 2000; 83: 2171-2178.

8. Deng Y, Ren X, Yang L. JNK-dependent pathway is required for TNFalpha-induced apoptosis. Cell 2003; 115: 61-70.

9. Dhanasekaran DN, Reddy EP. JNK signaling in apoptosis. Oncogene 2008; 27: 6245-6251.

10. Eckelman BP, Salvesen GS, Scott FL. Human inhibitor of apoptosis proteins: why XIAP is the black sheep of the family. EMBO Rep 2006; 7: 988-994.

11. GBD 2016 Traumatic Brain Injury and Spinal Cord Injury Collaborators. Global, regional, and national burden of traumatic brain injury and spinal cord injury, 1990-2016: a systematic analysis for the Global Burden of Disease Study 2016. Lancet Neurol 2019; 18: 56-87.
12. Glorieux C, Zamocky M, Sandoval JM, Verrax J, Calderon PB. Regulation of catalase expression in healthy and cancerous cells. Free Radic Biol Med 2015; 87: 84-97.

13. Hayes JP, Bigler ED, Verfaellie M. Traumatic brain injury as a disorder of brain connectivity. I Int Neuropsychol Soc 2016; 22: 120-137.

14. Hu W, Jiang J, Yang F, Liu J. The impact of bone marrow-derived mesenchymal stem cells on neovascularisation in rats with brain injury. Folia Neuropathol 2018; 56: 112-123.

15. Humphreys I, Wood RL, Phillips CJ, Macey S. The costs of traumatic brain injury: a literature review. Clinicoecon Outcomes Res 2013; 5: 281-287.

16. Jin Z, El-Deiry WS. Overview of cell death signaling pathways. Cancer Biol Ther 2005; 4: 139-163.

17. Kang JG, Park JS, Ko JH, Kim YS. Regulation of gene expression by altered promoter methylation using a CRISPR/Cas9-mediated epigenetic editing system. Sci Rep 2019; 9: 11960.

18. Kodydková J, Vávrová L, Kocík M, Žák A. Human catalase, its polymorphisms, regulation and changes of its activity in different diseases. Folia Biol (Praha) 2014; 60: 153-167.

19. Li Z, Hannigan M, Mo Z, Liu B, Lu W, Wu Y, Smrcka AV, Wu G, Li L, Liu M, Huang CK, Wu D. Directional sensing requires $\mathrm{G}$ beta gamma-mediated PAK1 and PIX alpha-dependent activation of Cdc42. Cell 2003; 114: 215-227.

20. Liu C, Vyas A, Kassab MA, You Z, Yu X. The role of poly ADP-ribosylation in the first wave of DNA damage response. Nucleic Acids Res 2017; 45: 8129-8141.

21. Llovet JM, Bruix J. Molecular targeted therapies in hepatocellular carcinoma. Hepatology 2008; 48: 1312-1327.

22. Lu J, Holmgren A. Thioredoxin system in cell death progression. Antioxid Redox Signal 2012; 17: 1738-1747.

23. Nobes CD, Hall A. Rho, rac, and cdc42 GTPases regulate the assembly of multimolecular focal complexes associated with actin stress fibers, lamellipodia, and filopodia. Cell 1995; 81: 53-62.

24. Olczak M, Poniatowski $Ł$, Kwiatkowska M, Samojłowicz D, Tarka S, Wierzba-Bobrowicz T. Immunolocalization of dynein, dynactin, and kinesin in the cerebral tissue as a possible supplemental diagnostic tool for traumatic brain injury in postmortem examination. Folia Neuropathol 2019; 57: 51-62.

25. Park JH, Kwon JG, Kim SJ, Song DK, Lee SG, Kim ES, Cho KB, Jang BI, Kim DH, Sin JI, Kim TW, Song IH, Park KS. Alterations of colonic contractility in an interleukin10 knockout mouse model of inflammatory bowel disease. J Neurogastroenterol Motil 2015; 21: 51-61.

26. Park L, Anrather J, Girouard H, Zhou P, Iadecola C. Nox2-derived reactive oxygen species mediate neurovascular dysregulation in the aging mouse brain. J Cereb Blood Flow Metab 2007; 27: 1908-1918.

27. Pfaffl MW. A new mathematical model for relative quantification in real-time RT-PCR. Nucleic Acids Res 2001; 29: e45.

28. Rakotoarisoa M, Angelov B, Espinoza S, Khakurel K, Bizien T, Angelova A. Cubic liquid crystalline nanostructures involving catalase and curcumin: BioSAXS study and catalase peroxidatic function after cubosomal nanoparticle treatment of differentiated SH-SY5Y cells. Molecules 2019; 24: E3058. 
29. Ramlackhansingh AF, Brooks DJ, Greenwood RJ, Bose SK, Turkheimer FE, Kinnunen KM, Gentleman S, Heckemann RA, Gunanayagam K, Gelosa G, Sharp DJ. Inflammation after trauma: microglial activation and traumatic brain injury. Ann Neurol 2011; 70: 374-383.

30. Schaible EV, Windschugl J, Bobkiewicz W, Kaburov Y, Dangel L, Krämer T, Huang C, Sebastiani A, Luh C, Werner C, Engelhard K, Thal SC, Schäfer MK. 2-Methoxyestradiol confers neuroprotection and inhibits a maladaptive HIF-1alpha response after traumatic brain injury in mice. J Neurochem 2014; 129: 940-954.

31. Sepasi Tehrani H, Moosavi-Movahedi AA. Catalase and its mys teries. Prog Biophys Mol Biol 2018; 140: 5-12.

32. Tanaka T, Hosoi F, Yamaguchi-Iwai Y. Thioredoxin-2 (TRX-2) is an essential gene regulating mitochondriadependent apoptosis. EMBO J 2002; 21: 1695-1703.

33. Tartaglia M, Pennacchio LA, Zhao C, Yadav KK, Fodale V, Sarkozy A, Pandit B, Oishi K, Martinelli S, Schackwitz W, Ustaszewska A, Martin J, Bristow J, Carta C, Lepri F, Neri C, Vasta I, Gibson K, Curry CJ, Siguero JP, Digilio MC, Zampino G, Dallapiccola B, Bar-Sagi D, Gelb BD. Gain-of-function SOS1 mutations cause a distinctive form of Noonan syndrome. Nat Genet 2007; 39: 75-79.

34. Wada K, Chatzipanteli K, Busto R, Dietrich WD. Role of nitric oxide in traumatic brain injury in the rat. J Neurosurg 1998; 89: 807-818.

35. Wang Z, Wei X, Liu K, Zhang X, Yang F, Zhang H, He Y, Zhu T, Li F, Shi W, Zhang Y, Xu H, Liu J, Yi F. NOX2 deficiency ameliorates cerebral injury through reduction of complexin IImediated glutamate excitotoxicity in experimental stroke. Free Radic Biol Med 2013; 65: 942-951.

36. Wiśnik E, Płoszaj T, Robaszkiewicz A. Downregulation of PARP1 transcription by promoter-associated E2F4-RBL2-HDAC1-BRM complex contributes to repression of pluripotency stem cell factors in human monocytes. Sci Rep 2017; 7: 9483.

37. Xu J, Shi J, Zhang J, Zhang Y. Vorinostat: a histone deacetylases (HDAC) inhibitor ameliorates traumatic brain injury by inducing iNOS/Nrf2/ARE pathway. Folia Neuropathol 2018; 56: 179-186.

38. Yamamoto K, Ichijo H, Korsmeyer SJ. BCL-2 is phosphorylated and inactivated by an ASK1/Jun N-terminal protein kinase pathway normally activated at G(2)/M. Mol Cell Biol 1999; 19: 8469-8478.

39. Yu F, Xue W, Dong L, Hu X, Huang D, Wang K. Tetrahydroxystilbene glucoside suppresses NAPDH oxidative stress to mitigate apoptosis and autophagy induced by cerebral ischemia/reperfusion injury in mice. Evid Based Complement Alternat Med 2019; 2019: 3913981

40. Zhang M, Sun L, Wang X, Chen S, Kong Y, Liu N, Chen Y, Jia O Zhang L, Zhang L. Activin B promotes BMSC-mediated cutaneous wound healing by regulating cell migration via the JNK-ERK signaling pathway. Cell Transplant 2014; 23: 1061-1073.

41. Ziaja M, Pyka J, Machowska A, Maslanka A, Plonka PM. Nitric oxide spin-trapping and NADPH-diaphorase activity in mature rat brain after injury. J Neurotrauma 2007; 24: 1845-1854. 